\title{
Review Article \\ The Role of Ultrafiltration in Patients with Decompensated Heart Failure
}

\author{
Sandeep A. Kamath \\ Division of Cardiovascular Medicine, University of Virginia Health System, P.O. Box 800158, Charlottesville, VA 22908, USA \\ Correspondence should be addressed to Sandeep A. Kamath, kamath@virginia.edu
}

Received 15 August 2010; Accepted 1 September 2010

Academic Editor: Mitchell H. Rosner

Copyright () 2011 Sandeep A. Kamath. This is an open access article distributed under the Creative Commons Attribution License, which permits unrestricted use, distribution, and reproduction in any medium, provided the original work is properly cited.

Congestion, due in large part to hypervolemia, is the primary driver of heart failure (HF) admissions. Relief of congestion has been traditionally achieved through the use of loop diuretics, but there is increasing concern that these agents, particularly at high doses, may be deleterious in the inpatient setting. In addition, patients with HF and the cardiorenal syndrome (CRS) have diminished response to loop diuretics, making these agents less effective at relieving congestion. Ultrafiltration, a mechanical volume removal strategy, has demonstrated promise in achieving safe and effective volume removal in patients with cardiorenal syndrome and diuretic refractoriness. This paper outlines the rationale for ultrafiltration in CRS and the available evidence regarding its use in patients with HF. At present, the utility of ultrafiltration is restricted to selected populations, but a greater understanding of how this technology impacts HF and CRS may expand its use.

\section{Introduction}

Despite significant advances in therapy and understanding of the disease, heart failure (HF) continues to remain a very morbid, mortal, and resource-consuming chronic illness. The total estimated direct and indirect cost of $\mathrm{HF}$ in the United States for 2010 is $\$ 39.2$ billion [1]. Of particular concern are patients admitted to the hospital with congestion, as these patients are at greater risk of morbidity and mortality than those with stable heart failure [2]. Mean 30 -day mortality in these patients is slightly above $10 \%$, and the mean 30-day readmission rate is around 30\% [3]. Dyspnea, a symptom of congestion and volume overload, is present in almost $90 \%$ of patients admitted to the hospital with heart failure [4]. Therapy aimed at relieving congestion and volume overload is therefore essential; prevention of recurrent volume accumulation is critical to disease stabilization. For over 50 years, the use of loop diuretics has been the main way to achieve fluid loss and decongestion. As heart failure progresses, patients may develop a declining renal function and a diuretic unresponsiveness, a condition termed the cardiorenal syndrome (CRS), which may make volume removal with diuretics difficult. It is in this setting that alternative means of fluid removal require consideration.
This paper focuses on a mechanical method of fluid removal known as ultrafiltration.

\section{Loop Diuretics: The Mainstay of Therapy for Volume Overload}

As mentioned above, the predominant reason that $\mathrm{HF}$ patients present to the hospital is due to symptoms of congestion. These symptoms are usually associated with venous congestion and volume overload. In addition, signs of elevated venous congestion, namely a third heart sound and jugular venous distention, portend a poor prognosis in HF patients [5]. It is critically important, therefore, to reduce venous congestion prior to the development of symptoms and also to quickly relieve symptoms of congestion once present. This has been accomplished almost exclusively via the use of loop diuretics since about 1965. Loop diuretics block the sodium-potassium-chloride transporter in the ascending limb of the Loop of Henle. In order to act, they must be secreted into the tubular lumen. The pharmacokinetics and pharmacodynamics of these agents can vary considerably from patient to patient, and therefore these agents must be titrated to effective doses while minimizing toxicity [6]. With over 40 years of clinical experience in 
using these agents, loop diuretics have been given a level A recommendation to restore and maintain normal volume status in HF patients in the Heart Failure Society of America practice guidelines [7], despite the availability of large-scale randomized trials which would be required to garner such a recommendation for a novel agent. There are problems with loop diuretics that have caused many to question their use, especially in the setting of decompensation.

\section{Diuretic Resistance}

Diuretic resistance, simply defined, is the progressive lack of efficacy of a given dose of diuretic to achieve an adequate urinary response. This necessitates the use of higher doses and combinations of loop and nonloop diuretics to achieve sodium and fluid loss, often at the expense of worsening renal function. Many factors contribute to the development of diuretic resistance. Worsening renal insufficiency leads to less secretion of diuretic into the tubular fluid, requiring a greater overall dose of diuretic in order for an effective amount of diuretic to reach its site of action [6]. Increased activation of the renin-angiotensin-aldosterone system, induced by diuretic use, results in increased sodium and water reabsorption through a variety of mechanisms. Hypertrophy of distal tubule epithelial cells results in greater sodium absorption distal to the Loop of Henle, the site of action of loop diuretics [8]. In patients with decompensated heart failure, venous pressure is also elevated, leading to decreased absorption of oral agents and decreased renal blood flow and renal sodium excretion [9]. Diuretic resistance is often found to coexist with renal insufficiency in patients with HF and, when present, defines the cardiorenal syndrome.

\section{Loop Diuretics May Be Harmful}

In addition to having diminished efficacy in patients with CRS, loop diuretics themselves, particularly when administered in high doses in an inpatient setting, may be harmful. Retrospective analyses of large multicenter trials of patients with HF have shown a consistent trend that nonpotassium sparing diuretics (NPSDs), of which loop diuretics are the primary agents used, are associated with worsened outcomes. An analysis of the Studies of Left Ventricular Dysfunction (SOLVD), a seminal randomized trial demonstrating the benefit of ACE inhibition in the progression of HF, demonstrated a higher all-cause and cardiovascular mortality in subjects on NPSDs versus those who were not [10]. These differences were not significant after multivariable adjustment, but a higher risk of arrhythmic death in patients on NPSDs persisted. An analysis of the Digitalis Investigation Group (DIG) trial, conducted to evaluate the influence of digoxin in mild-to-moderate chronic HF, also showed an increased risk of death, cardiovascular death, progressive HF death, sudden cardiac death, and HF hospitalizations in subjects on NPSDs [11, 12]. A subsequent study of over 1300 patients with advanced heart failure at a single center demonstrated a linear decrease in survival with increasing outpatient dose of loop diuretic [13]. This association persisted in multivariable analysis.
The Evaluation Study of Congestive Heart Failure and Pulmonary Artery Catheterization Effectiveness (ESCAPE) trial, conducted to evaluate the use of pulmonary artery catheter in subjects admitted to the hospital with advanced heart failure, found a similar dose-response relationship between inpatient loop diuretic dose and adjusted 6-month mortality [14]. Using the Acute Decompensated Heart Failure (ADHERE) national registry, a large nationwide database of patients admitted to the hospital with heart failure, investigators were able to demonstrate that subjects receiving an inpatient intravenous dose of less than $160 \mathrm{mg}$ of furosemide equivalents had lower in-hospital mortality, fewer episodes of worsening renal function, and shorter length of stay than subjects treated with $>160 \mathrm{mg}$ of furosemide equivalents per day, after propensity adjustment [15]. These association studies do not prove causation, and it is certainly possible that the need for a loop diuretic or a higher dose of loop diuretic is simply reflective of a greater HF disease severity and not an increased risk attributable to the loop diuretic itself. Small clinical trials have demonstrated short-term adverse clinical outcomes with higher doses of intravenous loop diuretics $[16,17]$. One mechanism through which loop diuretics may exert a negative prognostic influence is hypokalemia, which may lead to ventricular arrhythmias. Activation of the renin-angiotensin-alsoterone system and sympathetic nervous system [18-20], known to influence HF progression, has been postulated as another potential mechanism. In a porcine model of pacing-induced HF, furosemide administration shortened time to left ventricular dysfunction, and serum aldosterone levels were significantly higher in the furosemide-treated animals [21].

The recently presented NIH-sponsored Diuretic Optimization Strategies Evaluation (DOSE) study was the first randomized trial of diuretic therapy in heart failure. It randomized patients admitted with decompensated $\mathrm{HF}$ and high outpatient diuretic dose (between $80 \mathrm{mg}$ and $240 \mathrm{mg}$ of furosemide daily) to high doses (2.5 times oral dose) and low doses (equivalent oral dose) of furosemide as well as continuous infusion versus intermittent therapy in a $2 \times 2$ factorial design. The change in creatinine from baseline to 72 hours was low and not different among groups. In the high dose group, there was a higher rate of creatinine elevation $>3 \mathrm{mg} / \mathrm{dL}$, but this did not translate into any difference in 60 day outcomes. Sixty day rate of death, rehospitalization, or ED visit was not different among groups and approached 45$50 \%$. The study does provide some short-term data regarding the safety of intravenous loop diuretics in the inpatient setting. Lower dose diuretic therapy may be preferred as an initial approach, provided that the dose is escalated if there is suboptimal response at 48 hours.

\section{What Is Ultrafiltration and How Does It Differ from Hemodialysis?}

For nonnephrologists, it is useful to briefly review the underlying concepts of ultrafiltration (UF). UF involves a convective transfer of water and solutes (Figure 1). Plasma water is forced across a semipermeable membrane that allows movement of water and solutes (small molecules less 


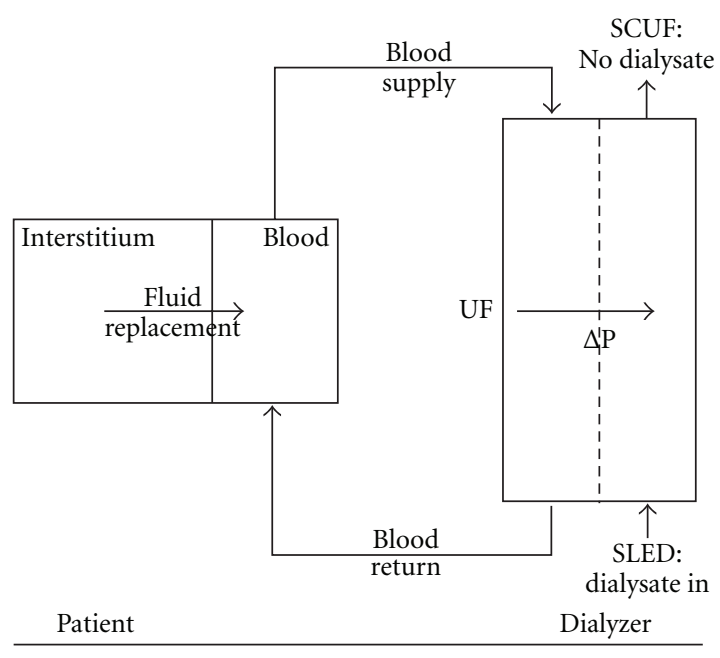

Figure 1: Dialytic techniques used in decompensated heart failure. Slow continuous ultrafiltration (SCUF) uses a hydrostatic pressure difference $(\Delta \mathrm{P})$ between the blood and nonblood sides of the membrane (dotted line within the dialyzer) to remove water and solutes from the plasma by ultrafiltration. Sustained low-efficiency dialysis (SLED) has the additional feature of dialysis fluid passed through the nonblood compartment in a countercurrent direction to the blood flow. Fluid removed from the blood must be replaced by transfer from the interstitial compartment. Failure of this fluid recovery will result in hemodynamic instability.

than $20 \mathrm{kDa}$ ) across the filter based on the transmembrane pressure difference $(\Delta \mathrm{P})$ between the blood and filtrate sides of the filter. Solute particles that are smaller than the filter pores can be "dragged" across into the ultrafiltrate with plasma water and are in the same concentration in the ultrafiltrate as they are in the prefilter plasma; thus the ultrafiltrate, or volume removed, is isotonic to plasma. The magnitude of water and solute clearance is proportional to the amount of ultrafiltrate formed and can be manipulated by changing the $\Delta \mathrm{P}$ (i.e., by increasing the blood flow or by applying suction to the filtrate side). In slow continuous UF, the approach favored in HF patients, the amount of ultrafiltrate created is small (generally $2-4 \mathrm{~mL} / \mathrm{minute}$ ) and does not require replacement fluid infusion. The higher the rate of ultrafiltrate formed, the greater the chance of causing hemoconcentration and intravascular volume depletion. The goal is to remove volume at the same rate it can be recovered from the extravascular space. UF is generally used when loss of plasma water (and not solute clearance) is the main goal of therapy [22]. As most patients with HF have no need for solute exchange, this is the preferred and most studied method for mechanical volume removal in $\mathrm{HF}$ patients. The development of lower flow UF systems not requiring conventional dialysis catheters but rather large bore IV catheters has also led to potential implementation by nonrenal physicians and staff, also making this technology more broadly applied.

Conversely, the primary purpose of hemodialysis (HD) is solute exchange, not volume removal. In $\mathrm{HD}$, solute transport occurs by passive diffusion and generally favors clearance of small molecules less than $\sim 300 \mathrm{Da}$ in size. The patient's blood and dialysate are separated by a semipermeable membrane with relatively small pores. Electrolytes and other solute particles small enough to pass through membrane pores diffuse freely down their concentration gradients, leading theoretically to equal concentrations on either side of the membrane. A process known as hemodiafiltration combines HD and UF by running dialysate countercurrent to blood flow and applying a pressure gradient across the membrane, yielding both diffusive and convective clearance. The large amount of ultrafiltrate created necessitates replacement fluid infusion.

\section{Clinical Trials of UF in Patients with HF}

Marenzi et al. studied the effects of UF in 24 patients with refractory $\mathrm{CHF}$ admitted to the cardiac intensive care unit for treatment of heart failure [23]. All had signs of volume overload. All patients were treated with UF via a conventional CRRT machine; access was via a double lumen $y$-shaped catheter in a femoral vein. UF resulted in an average of $4.9 \mathrm{~L}$ of fluid removal over a 9-hour period. Symptoms improved, and the response to subsequent diuretic therapy was enhanced, with a reduction in mean dose of diuretic following UF therapy. All patients had continuous hemodynamic data available via a Swann-Ganz catheter as well as invasive arterial pressure via an arterial line. No changes in heart rate, mean blood pressure, or systemic vascular resistance were observed, while mean right atrial pressure, pulmonary capillary wedge pressure, and mean pulmonary artery pressure were reduced. Intravascular volume, as estimated by hematocrit values, remained stable throughout the entire time of treatment despite the large amount of fluid removed overall. A fall in filling pressures with stable hematocit during UF indicated that a proportional volume of fluid was refilling the vasculature from the congested interstitium. This and other uncontrolled studies of UF in HF [24-26] showed that UF could be performed safely and could result in significant volume removal and symptom relief. These studies were performed using conventional renal dialysis equipment, but they led to the development of proprietary systems that were less cumbersome, lacked the need for central venous access, and required less specialized expertise to operate. In order to gain FDA approval for such equipment, randomized trials were required, which led to more robust data regarding the safety and efficacy of UF in patients with HF.

Costanzo et al. examined the utility of UF at a single center in 20 patients admitted with HF, volume overload, and renal insufficiency or diuretic resistance, defined as serum creatinine $\geq 1.5 \mathrm{mg} / \mathrm{dL}$ or furosemide $>80 \mathrm{mg} /$ day. Patients must not have had more than one diuretic dose prior to enrollment and must have been enrolled within 12 hours of admission. Major exlusion criteria were hematocrit $>40 \%$, systolic $\mathrm{BP}<85 \mathrm{mmHg}$, IV vasoactive therapy. Improvement in volume overload after ultrafiltration persisted at 30 and 90 days post discharge and no changes in renal function, electrolytes, or systolic BP were observed at hospital discharge, 30 days, or 90 days post discharge. Symptom scores improved by hospital discharge and these improvements were sustained 
at 30 and 90 days. Notably, in the 3 months preceding ultrafiltration, 10 hospitalizations occurred in 9 patients. After ultrafiltration, one patient was admitted within 30 days and two more were admitted between 30 and 90 days for unrelated causes (not complications of UF and not CHF); medications did not change significantly for the 20 patients [27]. This study showed promising durability of the fluid removal by UF in addition to the short-term gains seen in prior studies but was limited by the lack of a control group.

The Relief for Acutely fluid-overloaded Patients with decompensated CHF (RAPID-CHF) trial was the first clinical trial to test the use of a less invasive UF device (System 100, CHF Solutions, Brooklyn Park, MN) that used a single 16-g intravenous catheter in the antecubital fossa rather than central venous access like most conventional devices capable of UF. This study was unblinded. A total of 40 patients were enrolled at 6 sites and randomized $1: 1$ to usual care or UF plus usual care. Inclusion criteria were inpatient admission with primary diagnosis of CHF, 2+ lower extremity edema, and one other sign of increased congestion. Major exclusion criteria included severe stenotic valvular disease, acute coronary syndrome, systolic BP < $90 \mathrm{mmHg}$ at time of consent, hematocrit $>40 \%$, poor peripheral venous access, and severe concomitant disease. All patients in the UF plus usual care group received a single 8-hour course of UF with fluid removal rates determined by the attending physician (up to $500 \mathrm{cc} / \mathrm{hr}$ ). Diuretics were held during UF; thereafter, diuretics were administered at the discretion of the attending physician. Additional UF courses were allowed at the discretion of the treating physician. The primary endpoint was weight loss assessed at 24 hours after consent was obtained. In this intention-to-treat analysis, 2 patients in the UF group did not receive UF: one due to unsuccessful IV access and one due to inability to withdraw blood from the catheter. There was greater volume removal at 24 hours, but weight loss at 24 hours was not different between the two groups. Heart rate, systolic blood pressure, and electrolytes were not different between the two groups at 24 hours. Global dyspnea and CHF scores were improved in the UF group. UF was well tolerated without clinically significant bleeding or hypotension. There was one catheter site infection requiring a 4-week course of IV antibiotics [28].

The ultrafiltration versus intravenous diuretics for patients hospitalized for acute decompensated heart failure (UNLOAD) trial [29] enrolled a total of 200 patients at 28 centers. Patients were eligible if admitted to the hospital and enrolled within 24 hours with a primary diagnosis of heart failure and with 2 signs of hypervolemia. Exclusions were similar to RAPID-CHF, except that hematocrit had to be less than $45 \%$ and serum creatinine had to be at or below $3.0 \mathrm{mg} / \mathrm{dL}$. Study participants were randomized to usual care or usual care plus UF with the System 100 device (CHF Solutions, Brooklyn Park, MN). Total extracorporeal blood volume of this device is $33 \mathrm{~mL}$. All patients received $2 \mathrm{~g}$ sodium diet and $2000 \mathrm{~mL}$ fluid restriction. Subjects in the usual care group received a minimum intravenous diuretic of twice the before-hospitalization oral daily dose of diuretic. Subjects in the UF group received UF at up to $500 \mathrm{~mL} / \mathrm{hr}$ with duration and rate left to discretion of treating physician.

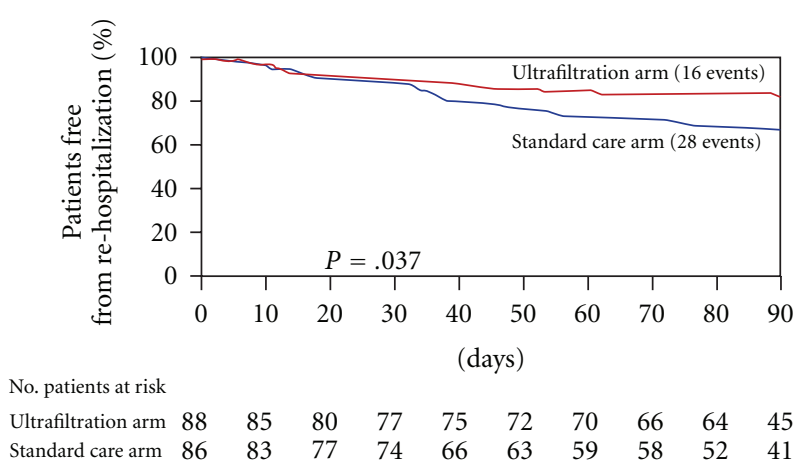

FIgURE 2: UNLOAD trial, freedom from rehospitalization. KaplanMeier estimate for freedom from rehospitalization for heart failure within 90 days of discharge in the ultrafiltration (red line) and usual care (blue line) groups.

Mean serum creatinine was $1.5 \mathrm{mg} / \mathrm{dL}$ in both groups; mean BNP was around $1300 \mathrm{pg} / \mathrm{mL}$; mean daily oral dose of loop diuretic (furosemide equivalents) prior to admission was $120 \mathrm{mg}$. Patients were followed for 90 days or until death. Twenty patients (10\%) died by 90 days, 9 in the UF group and 11 in the usual care goup; the study was not powered to detect differences in mortality. The trial met one of its primary efficacy endpoints of improved weight loss at 48 hours, but there was no difference in dyspnea score at 48 hours, the other primary efficacy endpoint. Dyspnea scores did not correlate with other HF-related outcomes. Fewer patients in the UF group required IV vasoactive therapies at 48 hours. With regard to safety, significant elevations in creatinine were similar in both groups; no correlation was found between fluid removed and changes in serum creatinine in either group. Hypotension during the $48 \mathrm{hr}$ period following randomization was similarly low in both groups. Fewer bleeding events occurred in the UF group than in the usual care group. Hypokalemia $(\mathrm{K}<3.5 \mathrm{mEq} / \mathrm{L})$ was less frequent in the UF group. With regard to secondary endpoints, lengths of stay were similar despite greater fluid loss in the UF group. Oral furosemide doses at discharge were lower in the UF group. Perhaps the most important observation in this study was the decrease in HF hospitalizations, HF rehospitalizations, rehospitalization days per patient, and unscheduled and emergency department visits for HF in the UF group (Figure 2). A subsequent analysis [30] demonstrated that this benefit was consistent relative to those treated with bolus or continuous infusion of intravenous diuretic.

Interestingly, there was a similar net fluid loss between subjects who received continuous infusion and those treated with UF, yet hospitalization rate was still lower in the UF group.

Liang et al. conducted a retrospective review of the experience at the Mayo Clinic using the System 100 device [31]. Patients in this small series had more advanced HF than in RAPID CHF and UNLOAD. A protocol had been developed prospectively in order to identify potential candidates for UF therapy. Ultrafiltration was attempted after failure of diuretic and/or IV vasoactive therapies. The case series included 11 patients with volume overload, systolic BP > $90 \mathrm{mmHg}$, 
and diuretic refractoriness (as per the discretion of treating physician). Three patients had constriction/restriction as the etiology of heart failure, 2 had ischemic cardiomyopathy, and none had nonischemic dilated cardiomypathy. Average serum creatinine was $2.2 \mathrm{mg} / \mathrm{dL}$ and average BUN was $69 \mathrm{mg} / \mathrm{dL}$. There were a total of 32 UF treatments that each lasted 8 hours in duration. Of the total UF runs, 75\% removed more than $2500 \mathrm{~mL}$ of fluid, and $41 \%$ removed $>3500 \mathrm{~mL}$. There were no serious bleeding complications. Notably, 5 out of 11 patients required dialysis on the same or subsequent admission and 6-month mortality was 55\%.

\section{Costs}

A recently published analysis using data derived from the UNLOAD study indicated that UF was associated with increased cost to society and the hospital versus IV diuretics, but decreased cost to Medicare via a decrease in hospitalizations for HF. The largest costs associated with UF were that of single-use disposable filters required for the proprietary UF system and hospital length of stay [32]. This analysis uses assumptions that tend to increase UF costs above that which might be seen in the real world, such as the use of multiple filters per patient and the use of UF (with similar length of stay and filter use) in a patient readmitted with HF who received UF during their index admission. Some have argued that using a conventional HD machine, which most hospitals already own, would reduce capital expenditure. Filter costs are also significantly lower for these machines. Currently, the disadvantages of such an approach, such as the need for central venous access and trained personnel and the lack of efficacy data supporting the use of conventional dialysis equipment in this manner, outweigh the potential savings [33, 34]. Future maneuvers by Medicare to incentivize hospitals to reduce readmission rates for HF may create a more favorable financial perspective for this technology from a hospital standpoint.

\section{Future Directions}

At this time, the reason for the increased efficacy of UF relative to diuretics is not clear. It does not appear to be entirely due to the amount of volume removed. Some have postulated that UF reduces levels of inflammatory cytokines, but this has not been proven; UF should not be able to clear such heavy molecules [35]. It is possible that relief of congestion, however it is achieved, will allow greater efficacy of loop diuretics and that UF is simply a more direct way to achieve this; the efficacy of UF is not dependent on renal function. Perhaps the removal of isotonic fluid with UF rather than hypotonic fluid with loop diuretics (i.e., total body sodium removal) is important [36]. Determining the mechanisms by which UF benefits HF patients, particularly those with CRS, may allow us to further elucidate the pathogenesis of CRS itself.

\section{Conclusion}

Congestion is the primary driver of admissions to the hospital due to HF. Relief of congestion has been traditionally achieved through the use of loop diuretics, but there is concern that these agents, particularly at high dose, may be deleterious in the inpatient setting. In addition, patients with advanced heart failure and the cardiorenal syndrome have diminished response to loop diuretics, making these agents less effective at relieving congestion. Preliminary data using UF, including a fairly large randomized trial, demonstrate no major safety concerns, improved volume removal versus diuretics alone, and decreased hospitalizations for $\mathrm{HF}$ at 90 days in selected patients. Major drawbacks are the increased cost of this technology and the invasiveness of the approach. Theoretical concerns, namely a predisposition to infectious and bleeding complications, especially due to the need for systemic heparinization during the procedure, have not been borne out in clinical trials. At the present time based on the available data, UF should be an inpatient therapeutic modality reserved as a second-line approach in diureticrefractory patients (well above $80 \mathrm{mg}$ /day of oral furosemide as outpatient and poor initial response to high-dose IV diuretics inpatient) with adequate blood pressure.

\section{References}

[1] D. Lloyd-Jones, R. J. Adams, T. M. Brown et al., "Heart disease and stroke statistics - 2010 update: a report from the american heart association," Circulation, vol. 121, no. 7, pp. e46-e215, 2010.

[2] A. Ahmed, R. M. Allman, G. C. Fonarow et al., "Incident f hospitalization and subsequent mortality in chronic Heart Failure: a propensity-matched study," Journal of Cardiac Failure, vol. 14, no. 3, pp. 211-218, 2008.

[3] G. K. Mulvey, Y. Wang, Z. Lin et al., "Mortality and readmission for patients with Heart Failure among U.S. News \& World Report's top heart hospitals," Circulation: Cardiovascular Quality and Outcomes, vol. 2, no. 6, pp. 558-565, 2009.

[4] K. F. Adams Jr., G. C. Fonarow, C. L. Emerman et al., "Characteristics and outcomes of patients hospitalized for Heart Failure in the United States: rationale, design, and preliminary observations from the first 100,000 cases in the Acute Decompensated Heart Failure National Registry (ADHERE)," American Heart Journal, vol. 149, no. 2, pp. 209216, 2005.

[5] M. H. Drazner, J. E. Rame, L. W. Stevenson, and D. L. Dries, "Prognostic importance of elevated jugular venous pressure and a third heart sound in patients with Heart Failure," The New England Journal of Medicine, vol. 345, no. 8, pp. 574-581, 2001.

[6] D. C. Brater, "Diuretic therapy," The New England Journal of Medicine, vol. 339, no. 6, pp. 387-395, 1998.

[7] J. Lindenfeld, N. M. Albert, J. P. Boehmer et al., "HFSA 2010 comprehensive Heart Failure practice guideline," Journal of Cardiac Failure, vol. 16, no. 6, pp. e1-e149, 2010.

[8] M. Sarraf, A. Masoumi, and R. W. Schrier, "Cardiorenal syndrome in acute decompensated Heart Failure," Clinical Journal of the American Society of Nephrology, vol. 4, no. 12, pp. 2013-2026, 2009.

[9] W. Mullens, Z. Abrahams, G. S. Francis et al., "Importance of venous congestion for worsening of renal function in advanced decompensated Heart Failure," Journal of the American College of Cardiology, vol. 53, no. 7, pp. 589-596, 2009.

[10] H. A. Cooper, D. L. Dries, C. E. Davis, Y. L. Shen, and M. J. Domanski, "Diuretics and risk of arrhythmic death in patients 
with left ventricular dysfunction," Circulation, vol. 100, no. 12, pp. 1311-1315, 1999.

[11] A. Ahmed, A. Husain, T. E. Love et al., "Heart Failure, chronic diuretic use, and increase in mortality and hospitalization: an observational study using propensity score methods," European Heart Journal, vol. 27, no. 12, pp. 1431-1439, 2006.

[12] M. Domanski, X. Tian, M. Haigney, and B. Pitt, "Diuretic use, progressive Heart Failure, and death in patients in the DIG study," Journal of Cardiac Failure, vol. 12, no. 5, pp. 327-332, 2006.

[13] S. Eshaghian, T. B. Horwich, and G. C. Fonarow, "Relation of loop diuretic dose to mortality in advanced Heart Failure," American Journal of Cardiology, vol. 97, no. 12, pp. 1759-1764, 2006.

[14] V. Hasselblad, W. G. Stough, M. R. Shah et al., "Relation between dose of loop diuretics and outcomes in a Heart Failure population: results of the ESCAPE Trial," European Journal of Heart Failure, vol. 9, no. 10, pp. 1064-1069, 2007.

[15] W. F. Peacock, M. R. Costanzo, T. De Marco et al., "Impact of intravenous loop diuretics on outcomes of patients hospitalized with acute decompensated Heart Failure: insights from the ADHERE registry," Cardiology, vol. 113, no. 1, pp. 12-19, 2009.

[16] G. Cotter, E. Metzkor, E. Kaluski et al., "Randomised trial of high-dose isosorbide dinitrate plus low-dose furosemide versus high-dose furosemide plus low-dose isosorbide dinitrate in severe pulmonary oedema," The Lancet, vol. 351, no. 9100, pp. 389-393, 1998.

[17] G. Cotter, J. Weissgarten, E. Metzkor et al., "Increased toxicity of high-dose furosemide versus low-dose dopamine in the treatment of refractory congestive Heart Failure," Clinical Pharmacology and Therapeutics, vol. 62, no. 2, pp. 187-193, 1997.

[18] J. Bayliss, M. Norell, and R. Canepa-Anson, "Untreated Heart Failure: clinical and neuroendocrine effects of introducing diuretics," British Heart Journal, vol. 57, no. 1, pp. 17-22, 1987.

[19] G. S. Francis, C. Benedict, D. E. Johnstone et al., "Comparison of neuroendocrine activation in patients with left ventricular dysfunction with and without congestive Heart Failure. A substudy of the studies of left ventricular dysfunction (SOLVD)," Circulation, vol. 82, no. 5, pp. 1724-1729, 1990.

[20] G. S. Francis, R. M. Siegel, and S. R. Goldsmith, "Acute vasoconstrictor response to intravenous furosemide in patients with chronic congestive Heart Failure. Activation of the neurohumoral axis," Annals of Internal Medicine, vol. 103, no. 1, pp. 1-6, 1985.

[21] J. M. McCurley, S. U. Hanlon, S.-K. Wei, E. F. Wedam, M. Michalski, and M. C. Haigney, "Furosemide and the progression of left ventricular dysfunction in experimental Heart Failure," Journal of the American College of Cardiology, vol. 44, no. 6, pp. 1301-1307, 2004.

[22] A. R. Nissenson, J. S. Berns, and E. V. Lerma, Current Diagnosis \& Treatment. Nephrology \& Hypertension, McGraw-Hill, New York, NY, USA, 2009.

[23] G. Marenzi, G. Lauri, M. Grazi, E. Assanelli, J. Campodonico, and P. Agostoni, "Circulatory response to fluid overload removal by extracorporeal ultrafiltration in refractory congestive Heart Failure," Journal of the American College of Cardiology, vol. 38, no. 4, pp. 963-968, 2001.

[24] G. Marenzi, S. Grazi, F. Giraldi et al., "Interrelation of humoral factors, hemodynamics, and fluid and salt metabolism in congestive Heart Failure: effects of extracorporeal ultrafiltration," American Journal of Medicine, vol. 94, no. 1, pp. 49-56, 1993.
[25] A. Rimondini, C. M. Cipolla, P. D. Bella et al., "Hemofiltration as short-term treatment for refractory congestive heart failure," American Journal of Medicine, vol. 83, no. 1, pp. 4348, 1987.

[26] I. A. Simpson, A. P. Rae, and K. Simpson, "Ultrafiltration in the management of refractory congestive Heart Failure," British Heart Journal, vol. 55, no. 4, pp. 344-347, 1986.

[27] M. R. Costanzo, M. Saltzberg, J. O’Sullivan, and P. Sobotka, "Early ultrafiltration in patients with decompensated Heart Failure and diuretic resistance," Journal of the American College of Cardiology, vol. 46, no. 11, pp. 2047-2051, 2005.

[28] B. A. Bart, A. Boyle, A. J. Bank et al., "Ultrafiltration versus usual care for hospitalized patients with Heart Failure: the relief for acutely fluid-overloaded patients with decompensated congestive Heart Failure (RAPID-CHF) trial," Journal of the American College of Cardiology, vol. 46, no. 11, pp. 20432046, 2005.

[29] M. R. Costanzo, M. E. Guglin, M. T. Saltzberg et al., "Ultrafiltration Versus Intravenous Diuretics for Patients Hospitalized for Acute Decompensated Heart Failure," Journal of the American College of Cardiology, vol. 49, no. 6, pp. 675683, 2007.

[30] M. R. Costanzo, M. T. Saltzberg, M. Jessup, J. R. Teerlink, and P. A. Sobotka, "Ultrafiltration is associated with fewer rehospitalizations than continuous diuretic infusion in patients with decompensated Heart Failure: results from UNLOAD," Journal of Cardiac Failure, vol. 16, no. 4, pp. 277-284, 2010.

[31] K. V. Liang, A. R. Hiniker, A. W. Williams, B. L. Karon, E. L. Greene, and M. M. Redfield, "Use of a novel ultrafiltration device as a treatment strategy for diuretic resistant, refractory Heart Failure: initial clinical experience in a single center," Journal of Cardiac Failure, vol. 12, no. 9, pp. 707-714, 2006.

[32] S. M. Bradley, W. C. Levy, and D. L. Veenstra, "Costconsequences of ultrafiltration for Acute Heart Failure: a decision model analysis," Circulation, vol. 2, no. 6, pp. 566573, 2009.

[33] A. Kazory, A. A. Ejaz, and E. A. Ross, "Ultrafiltration for Heart Failure: how fast should we move?" American Heart Journal, vol. 157, no. 2, pp. 205-207, 2009.

[34] E. A. Ross and A. Kazory, "Overcoming financial constraints of ultrafiltration for Heart Failure," American Journal of Cardiology, vol. 105, no. 10, pp. 1504-1505, 2010.

[35] A. Kazory and E. A. Ross, "Contemporary trends in the pharmacological and extracorporeal management of Heart Failure: a nephrologic perspective," Circulation, vol. 117, no. 7, pp. 975-983, 2008.

[36] B. A. Bart, "Treatment of congestion in congestive Heart Failure: ultrafiltration is the only rational initial treatment of volume overload in decompensated Heart Failure," Circulation: Heart Failure, vol. 2, no. 5, pp. 499-504, 2009. 


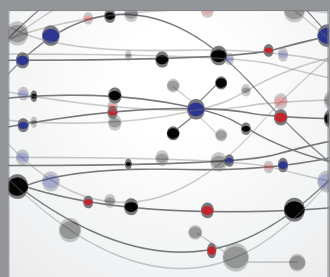

The Scientific World Journal
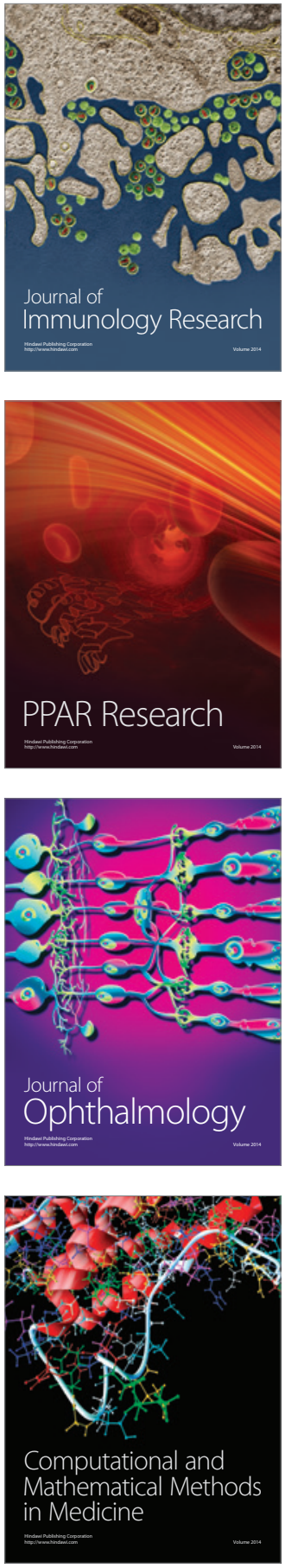

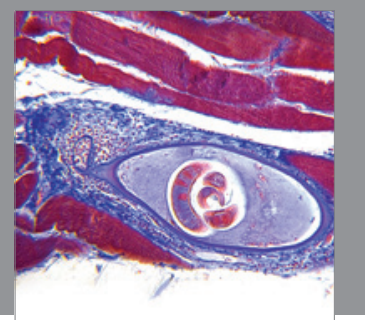

Gastroenterology

Research and Practice
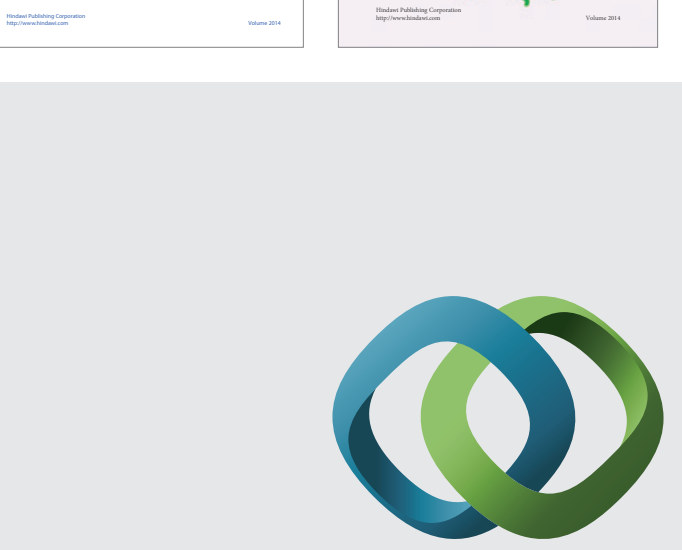

\section{Hindawi}

Submit your manuscripts at

http://www.hindawi.com
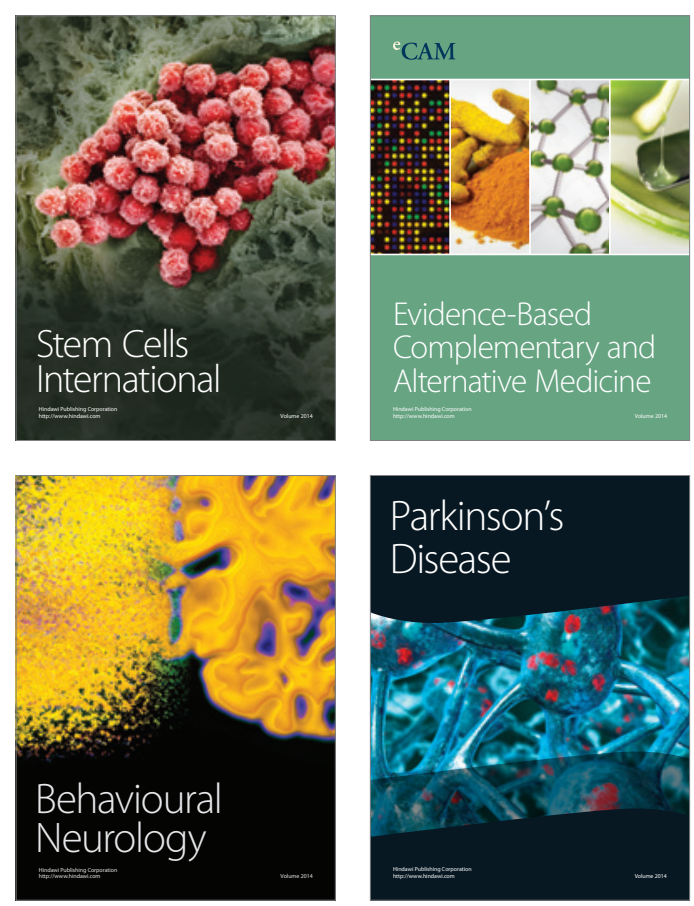

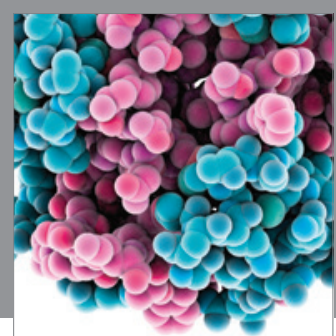

Journal of
Diabetes Research

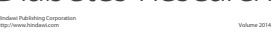

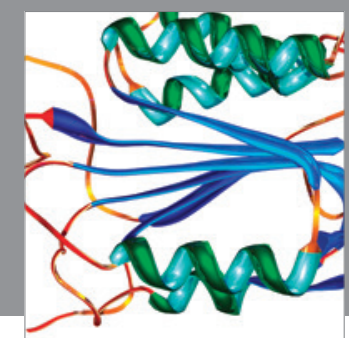

Disease Markers
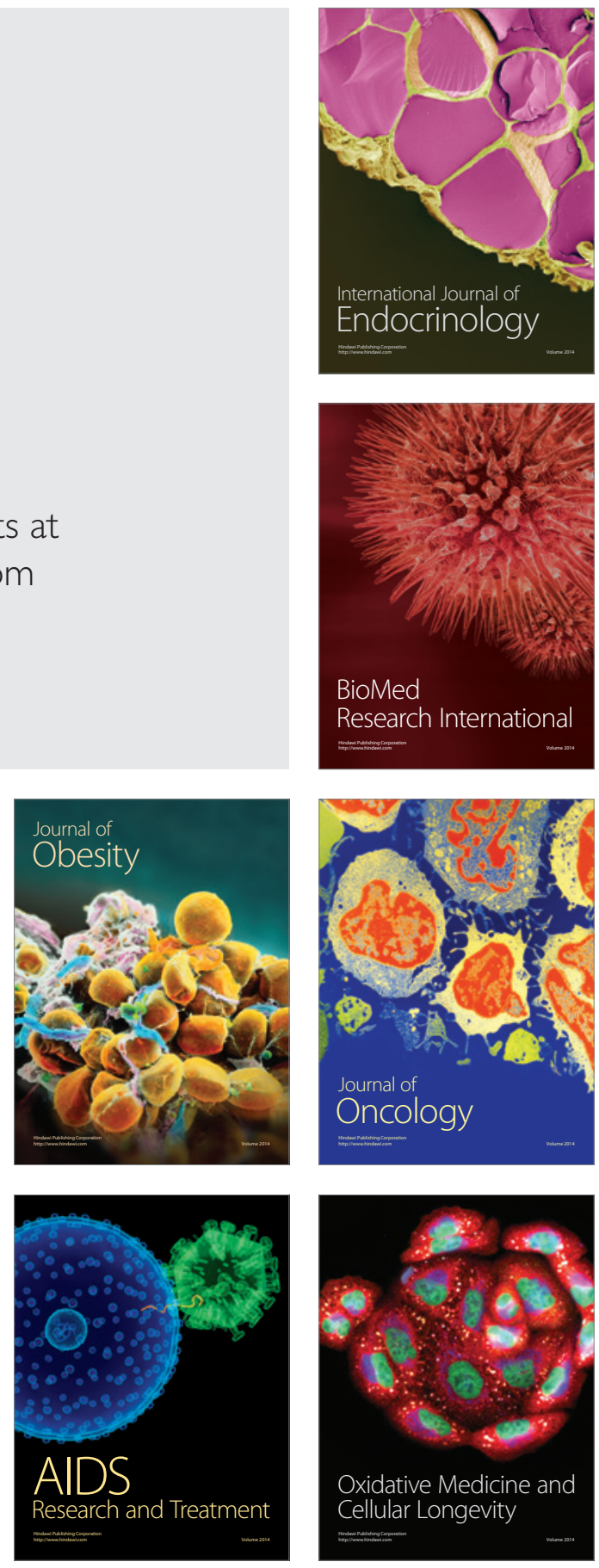